MATHEMATICS OF COMPUTATION

Volume 73, Number 245, Pages 127-142

S $0025-5718(03) 01548-5$

Article electronically published on June 6, 2003

\title{
DISCRETE ABSORBING BOUNDARY CONDITIONS FOR SCHRÖDINGER-TYPE EQUATIONS. PRACTICAL IMPLEMENTATION
}

\author{
ISAÍAS ALONSO-MALLO AND NURIA REGUERA
}

\begin{abstract}
Recently, some absorbing boundary conditions for Schrödingertype equations have been studied by Fevens, Jiang and Alonso-Mallo, and Reguera. These conditions make it possible to obtain a very high absorption at the boundary avoiding the nonlocality of transparent boundary conditions. However, the implementations used in the literature, where the boundary condition is chosen in a manual way in accordance with the solution or fixed independently of the solution, are not practical because of the small absorption. In this paper, a new practical adaptive implementation is developed that allows us to obtain automatically a very high absorption.
\end{abstract}

\section{INTRODUCTION}

Let us consider the initial value problem for a Schrödinger-type equation given by

$$
\left\{\begin{aligned}
\partial_{t} u(x, t) & =\frac{-i}{c}\left(\partial_{x x} u(x, t)+V u(x, t)\right), \quad x \in \mathbb{R}, \quad t \geq 0, \\
u(x, 0) & =u_{0}(x), \quad x \in \mathbb{R},
\end{aligned}\right.
$$

where $c>0$ is a real constant (the case $c<0$ is analogous). The study of (1.1) arises in a wide variety of applications, for example, the one-dimensional time dependent Schrödinger equation for a particle with mass $m$ [16] and the Fresnel equation for the evolution of a paraxial electrical field $E$ along the $z$-direction in a Cartesian coordinate system [13], 19].

For its numerical approximation, it is necessary to use artificial boundary conditions in order to work in a finite domain $\left[x_{l}, x_{r}\right] \times \mathbb{R}^{+}$. When, for $x \notin\left(x_{l}, x_{r}\right)$, the initial value $u_{0}(x)$ vanishes and the potential $V$ is constant, transparent boundary conditions (TBC) for (1.1) have been studied in [5], 6], [14, 20]. With these TBC, the solution in the computational subdomain coincides with the original solution. However, the main disadvantage of these TBC is that they are nonlocal in time, although some recent papers study algorithms for the fast evaluation of TBC [4], 14].

Received by the editor November 20, 2001 and, in revised form, May 7, 2002.

2000 Mathematics Subject Classification. Primary 65M12, 65M20; Secondary 65M99.

Key words and phrases. Schrödinger equation, transparent boundary conditions, absorbing boundary conditions.

The authors have obtained financial support from MCYT BFM 2001-2013 and JCYL VA025/01. 
Another possibility is to consider local absorbing boundary conditions (ABC), allowing small reflections at the interior of the computational window. These ABC have been studied in [1], [2], [3], [6], [8]. The main idea is to absorb the components of the solution traveling with certain velocities. For this, rational interpolatory functions approximating the symbol of the TBC in Fourier variables are used. With these $\mathrm{ABC}$, and if we know a priori the velocity of the solution, it is possible to obtain a very high absorption, comparable to that obtained with TBC [20].

In 2] the authors have proved that these $\mathrm{ABC}$ are weakly ill-posed and are worse posed when they are more absorbing. That explains why some numerical experiments show a bad behavior when the initial value does not vanish at the boundary of the computational domain (see [8]) or when the $\mathrm{ABC}$ is not suitable to absorb the solution of (1.1).

As an alternative, the authors have constructed in [3] other ABC (denoted by SABC) for a spatial semidiscrete version of (1.1) in a similar spirit to that of [9], proving that the semidiscrete problems obtained with these SABC present a similar weak ill-posedness. However, it is possible to obtain the same absorption with a greater value of the parameter of the spatial discretization. Therefore the influence of the weak ill-posedness is smaller.

Since the velocity of propagation of the solution is in general not known a priori, a practical implementation of these SABC must absorb all possible velocities. We remark that [2], 3, 8] do not provide this, although this fact is crucial to avoid the possible instability. A possibility is to use an implementation similar to that used in [6], [7], [11, where rational functions that interpolate the Fourier symbol of TBC at several fixed nodes are considered. The nodes are chosen in such a way that the rational function is an approximation of the Fourier symbol in an $L^{2}$ sense so that they are necessarily distinct. These authors use a partial fraction decomposition of the rational interpolatory function. We prove in $\S 2$ of this paper the existence and uniqueness of the interpolatory rational function of the Fourier symbol of TBC for the semidiscrete problem. Moreover, we show in $\S 3$ that the semidiscrete problems obtained with this implementation are also weakly ill-posed. Since there is no absorption for some velocities, some errors appear that lead to instability because of the weak ill-posedness. Therefore, this implementation is useless in practice.

To avoid the previous problems, we consider in $\S 4$ the possibility of using a few nodes, not necessarily distinct, but chosen with an adaptive strategy depending on the solution arriving at the boundary at each time step. Of course, any adaptive implementation is more cumbersome than the corresponding nonadaptive case, but in this case the adaptation is completely necessary because of the weak ill-posedness. The implementation of this strategy is carried out using Prony's method [15] to compute an approximation of the solution near the boundary as a sum of plane waves and sharing out the available interpolatory nodes in a suitable way in order to obtain the maximum absorption. Then, when a very high absorption is obtained, the weak ill-posedness is compensated for the high absorption, as is seen in [2], [3]. However, we note that when the absorption is not sufficient, the weak ill-posedness may emerge causing instabilities. Several numerical experiments confirm the results previously obtained.

Finally, we also remark that this adaptive implementation may be useful for the implementation of absorbing boundary conditions of other equations [7], 11], [12], for which, as far as we know, any adaptive technique has still been used. 


\section{AbSORBIng Boundary CONDITIONS}

We study in this section the absorbing boundary conditions for the spatial discretization of the initial value problem (1.1). Let $\left\{x^{j}\right\}_{j \in \mathbb{Z}}$ be a uniform mesh of $\mathbb{R}$, with $x^{j}=x_{l}+j h$, and denote by $u^{j}(t)$ an approximation of $u\left(x^{j}, t\right)$. For the spatial discretization of (1.1), we consider

$$
\begin{aligned}
\frac{d}{d t} u^{j} & =\frac{-i}{c}\left(\frac{u^{j+1}-2 u^{j}+u^{j-1}}{h^{2}}+V u^{j}\right), \quad j \in \mathbb{Z}, \quad t \geq 0, \\
u^{j}(0) & =u_{0}\left(x^{j}\right), \quad j \in \mathbb{Z} .
\end{aligned}
$$

We first state the transparent boundary conditions obtained in [2] for the discrete equation (2.1).

Theorem 2.1 (Transparent boundary conditions). Let $\left\{u^{j}(t)\right\}_{j \in \mathbb{Z}}$ be the solution of (2.1). Then, when $h^{2}(V+c \omega) / 2 \notin(0,2), \hat{u}^{j}(\omega)=0, j \in \mathbb{Z}$, and when $h^{2}(V+$ $c \omega) / 2 \in(0,2)$,

$$
\begin{aligned}
\hat{u}^{N-1}(\omega) & =r(V+c \omega, h) \hat{u}^{N}(\omega), \\
\hat{u}^{1}(\omega) & =r(V+c \omega, h) \hat{u}^{0}(\omega),
\end{aligned}
$$

where

$$
r(s, h)=1-\frac{h^{2}}{2} s+i \sqrt{\frac{h^{2}}{2} s\left(2-\frac{h^{2}}{2} s\right)} .
$$

The TBC (2.3) and (2.4) are nonlocal and we consider here the use of local SABC. For this, we approximate $r(s, h)$, given by (2.5), by means of interpolatory rational functions $q(s, h)$, for $s$ in the interval $\left(0,4 / h^{2}\right)$. When we consider a rational function $q=p_{1} / p_{2}$, with $p_{1}$ and $p_{2}$ relatively prime polynomials in $s$ with degrees $j_{1}$ and $j_{2}$, respectively, $\operatorname{SABC}\left(j_{1}, j_{2}\right)$ denotes the boundary conditions obtained, following the notation in [3]. Moreover, we also call the order of absorption the number $j_{1}+j_{2}+1$.

The second step is to choose several interpolatory nodes in the interval $\left(0,4 / h^{2}\right)$. Of course, the choice of these interpolatory nodes is crucial in order to obtain a practical implementation of these ABC. Let us consider a wave solution

$$
\left\{u^{j}(t)\right\}_{j \in \mathbb{Z}}=\{\exp (i(\eta j-\omega(\eta) t))\}_{j \in \mathbb{Z}}
$$

of the problem discretized in space, where $\omega(\eta)$ is given by the dispersion relation of (2.1)

$$
\omega(\eta)=\frac{2}{c h^{2}}(\cos (\eta)-1)+\frac{V}{c} .
$$

Therefore, if we want to absorb the wave solution (2.6) at the boundary, we should choose the interpolatory nodes in such a way that $r(V+c \omega, h)-q(V+c \omega, h)$ is small when $\omega=-\omega(\eta)$, that is, when $V+c \omega=\left(2 / h^{2}\right)(1-\cos \eta)$. That is to say, the approximation should be good when at least one of the interpolatory nodes is

$$
s_{1}=\frac{2}{h^{2}}(1-\cos (\eta)) \text {. }
$$

In practice, with this choice of the interpolatory nodes we can get to absorb the components of a general solution traveling with the group velocity of (2.6) (cf. [8]). 
The possible implementations considered below use several interpolatory nodes. Since the problem of the rational interpolation does not have existence and uniqueness in general, it is necessary to prove these facts for the function $r(s, h)$ given by $(2.5)$.

Theorem 2.2. Let $p_{1}$ and $p_{2}$ be relatively prime polynomials in $s$ with degrees $j_{1}$ and $j_{2}$, respectively. Let $s_{1}, s_{2}, \ldots, s_{j_{1}+j_{2}+1}$ be a set of interpolatory nodes in the interval $\left(0,4 / h^{2}\right)$. Then there exists a unique rational function $q(s, h)$ of exact type $\left(j_{1}, j_{2}\right)$ such that

$$
r\left(s_{j}, h\right)=q\left(s_{j}, h\right), \quad j=1, \ldots, j_{1}+j_{2}+1 .
$$

Proof. We suppose that $j_{1}=j_{2}+l$ with $l \geq 0$ (the case $j_{2} \geq j_{1}$ is similar). Then, we begin making two consecutive changes of variables. First, we make

$$
1-h^{2} s / 2=\cos (\theta), \quad \theta \in(0, \pi),
$$

(the case $\theta \in(\pi, 2 \pi)$ is similar). Now, the problem is to compute the rational function $\widetilde{q}(\theta):=q\left(2(1-\cos (\theta)) / h^{2}, h\right)$ which interpolates to the function $\exp (i \theta)$ at the nodes $\theta_{j}=\arccos \left(1-h^{2} s_{j} / 2\right), j=1, \ldots, j_{1}+j_{2}+1$ (notice that $\widetilde{q}(\theta)$ is independent of $h$ since $r(s, h)$ may be viewed as a function of $\left.h^{2} s / 2\right)$. Secondly, we make

$$
\tan (\theta / 2)=t, \quad t \in(0,+\infty)
$$

and the problem is now to compute the rational function $\bar{q}(t)=q\left(4 t^{2} /\left(h^{2}\left(1+t^{2}\right)\right), h\right)$ which interpolates the function $(1+i t) /(1-i t)$ at the points $t_{j}=\tan \left(\theta_{j} / 2\right), j=$ $1, \ldots, j_{1}+j_{2}+1$. That is to say, the equality

$$
\frac{1+i t}{1-i t}=\frac{p_{1}\left(\frac{4}{h^{2}} \frac{t^{2}}{1+t^{2}}\right)}{p_{2}\left(\frac{4}{h^{2}} \frac{t^{2}}{1+t^{2}}\right)},
$$

is satisfied for each one of the above points $t=t_{j}$. Equivalently, we have the equality

$$
\frac{1+i t}{1-i t}=\frac{\left(1+t^{2}\right)^{j_{1}} p_{1}\left(\frac{4}{h^{2}} \frac{t^{2}}{1+t^{2}}\right)}{\left(1+t^{2}\right)^{j_{1}} p_{2}\left(\frac{4}{h^{2}} \frac{t^{2}}{1+t^{2}}\right)}=\frac{q_{1}\left(t^{2}\right)}{q_{2}\left(t^{2}\right)},
$$

where both $q_{1}$ and $q_{2}$ are polynomial functions of degree $j_{1}$. A straightforward calculation leads to the equality

$$
t=\frac{i\left(q_{2}\left(t^{2}\right)-q_{1}\left(t^{2}\right)\right)}{q_{1}\left(t^{2}\right)+q_{2}\left(t^{2}\right)}=\frac{r_{1}\left(t^{2}\right)}{r_{2}\left(t^{2}\right)}
$$

for each $t=t_{1}, \ldots, t_{j_{1}+j_{2}+1}$. Then, $r_{1} / r_{2}$ is a rational function that interpolates $t^{1 / 2}$. We deduce from Theorem 8.1 in [10] that this problem has a unique solution. Undoing the two previous changes of variables, we obtain the wanted interpolatory rational function $q(s, h)$.

Although Theorem 2.2 covers other possibilities, in this paper we only consider the cases $j_{1}=j_{2}$ and $j_{1}=j_{2}+1$. In fact, the results in [3] show that other cases may present numerical instabilities. This behavior is similar to the one obtained for the continuous case in [2] for Schrödinger-type equations, in [10] for diffusion 
equations and in [11] for wave equations. Moreover, the stability of the simplest cases $\left(j_{1}, j_{2}\right)=(1,0)$ and $\left(j_{1}, j_{2}\right)=(1,1)$ is studied in [3], [17, [18.

Let us see how $\operatorname{SABC}(1,0)$ is obtained. For this, let us consider the polynomial $q(s, h)=\alpha_{0}+\alpha_{1} s$ that interpolates $r(s, h)$ at $s_{1}, s_{2}$. Then

$$
\alpha_{0}=1+\frac{2 i t_{1} t_{2}}{t_{1}+t_{2}}, \quad \alpha_{1}=\frac{i\left(t_{1}-i\right)\left(t_{2}-i\right)}{t_{1}+t_{2}},
$$

where $t_{j}$ and $s_{j}$ are related through the changes of variables (2.9) and (2.10).

Considering the approximation $r(s, h) \approx q(s, h)$ in $(2.3)$ and taking the inverse Fourier transform, we develop $\operatorname{SABC}(1,0)$,

$$
\frac{d}{d t} v^{N}(t)=\alpha v^{N}(t)+\beta v^{N+1}(t)
$$

with

$$
\alpha=\frac{\alpha_{0}+\alpha_{1} V}{i c \alpha_{1}}, \quad \beta=\frac{-1}{i c \alpha_{1}} .
$$

We have a similar expression for the left boundary condition, and we obtain in this way a first order system of ordinary differential equations

$$
u_{h}^{\prime}(t)=M(h) u_{h}(t),
$$

with $u_{h}(t)=\left[u^{0}, \ldots u^{N}\right]^{T}$.

Other SABC can be obtained in a similar way (see [3]). In the appendix we present the coefficients of the rational functions in some cases.

\section{NonAdAPtive IMPLEMENTATION}

We consider in this section the implementation of SABC for the equation (2.1) when fixed interpolatory nodes are chosen. We propose an implementation similar to that used in [6] for the continuous Schrödinger problem and 7], 11] for other problems. We consider approximations to $r(s, h)$, given by (2.5), by partial fractions,

$$
r(s, h) \approx q(s, h)=a_{0} x+\beta+\sum_{j=1}^{m} \frac{a_{j} x}{x+d_{j}},
$$

where $x=h^{2} s / 2$ and $q(s, h)$ interpolates $r(s, h)$ at $2 m+2$ different nodes $(2 m+1$ if $\left.a_{0}=0\right)$. We will denote the $\mathrm{ABC}$ obtained this way: $\mathrm{PF}(m+1, m)$ if $a_{0} \neq 0$ and $\operatorname{PF}(m, m)$ if $a_{0}=0$. For example, let us consider $m=1$ and let us use the approximation (3.1) in $\mathrm{TBC}(2.3)$ to develop an $\mathrm{ABC}$ of order four $\mathrm{PF}(2,1)$. Then, defining

$$
\frac{1}{h^{2}(V+c \omega) / 2+d_{1}} \hat{u}^{N}=\hat{\phi}
$$

we obtain

$$
\begin{aligned}
\frac{d}{d t} \phi & =\beta_{0} u^{N}+\beta_{1} \phi, \\
\frac{d}{d t} u^{N} & =\gamma_{0} u^{N-1}+\gamma_{1} u^{N}+\gamma_{2} \phi,
\end{aligned}
$$

for certain coefficients depending on $h$ and on the interpolatory nodes. We have a similar expression for the left boundary condition, for which we should define a function $\psi(t)$ analogous to $\phi(t)$. We obtain in this way a system similar to (2.11), with $u_{h}(t)=\left[\psi, u^{0}, \ldots u^{N}, \phi\right]^{T}$. Other ABC of higher order can be developed in 


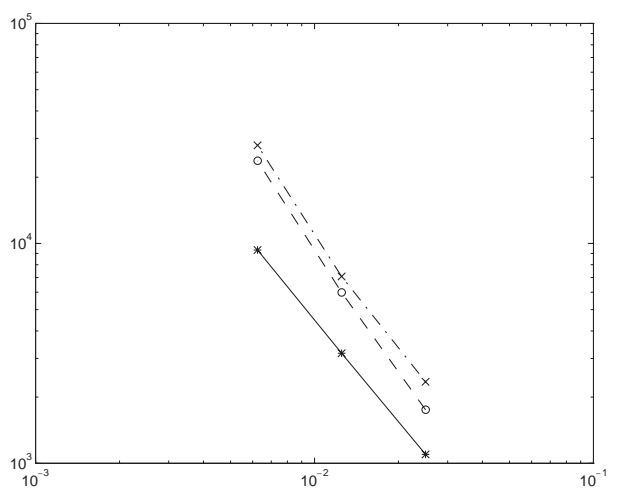

Figure 1. Condition number $\kappa_{h}$ as a function of $h$ : $-*$ for $\operatorname{PF}(2,1)$; $--\circ$ for $\operatorname{PF}(3,2)$; and $-\cdot-\times$ for $\operatorname{PF}(4,3)$.

the same way. The study of the well-posedness of these semidiscrete systems can be done similarly as in [2, 3]. We have checked numerically that the matrices of these systems have eigenvalues with negative real part. Therefore, $u_{h}(t) \rightarrow 0$ when $t \rightarrow+\infty$. Nevertheless, since these matrices are nonnormal, there could be an initial growth of the solution. The study of this initial possible bad behavior can be done as in [2], 3], analyzing the condition number $\kappa_{h}$ of the matrix $L(h)$ such that $M(h)=L(h) D(h) L(h)^{-1}$, where $D(h)$ is the diagonal matrix of eigenvalues. In Figure 1 we observe that $\kappa_{h}$ grows approximately as $O\left(h^{-2}\right)$ for the different ABC considered. Therefore, we see an advantage to this implementation. Although the semidiscrete systems are weakly ill-posed, this bad behavior does not seem to be worse for high order ABC. Nevertheless, we show in this section that the absorption with this implementation can be small and in fact not enough to compensate for this ill-posedness.

The choice of the interpolatory nodes in an interval $(0, \rho)$ should be done in order to minimize in some way the error committed when we approximate $r(s, h)$ by $q(s, h)$. Possible elections are Padé or the nodes that minimize the $L^{2}$ norm of this error in $(0, \rho)$. Nevertheless, the Padé nodes are not a good choice if the solution consists of several components traveling with different velocities, as is well showed in 3]. The choice of the nodes minimizing the $L^{2}$ norm has given good results in other problems (see [7, [11]) and this is the option we choose in this section. More precisely, we consider the rational function $q(s, h)$ given by (3.1) that interpolates $r(s, h)$ at the nodes $s_{j}, j=1, \ldots, 2 m+2$. These nodes are chosen so that

$$
\int_{0}^{\rho}|r(s, h)-q(s, h)|^{2} d s
$$

is minimum among all the rational functions of this kind. We remark that we perform this multivariate minimization by using routines of the NAG library, instead of considering a sequence of univariate minimizations as is done in [6], [11].

Another difficult choice is that of $\rho$. Since the interval of values for $s$ is $\left(0,4 / h^{2}\right)$, we could choose $\rho=4 / h^{2}$. Nevertheless, for $h$ small, this interval is too big and then, the interpolatory nodes are too separated. Therefore, the absorption of the velocities corresponding to values of $s$ different from the interpolatory nodes can 
be too small. It seems that we could choose $\rho \ll 4 / h^{2}$ but then, the velocities associated to values of $s$ bigger than $\rho$ will not be absorbed. These remarks can be observed in the following experiments. We have integrated the Fresnel equation

$$
2 i n_{0} k_{0} \partial_{z} E=\partial_{x}^{2} E+\left(n^{2}-n_{0}^{2}\right) k_{0}^{2} E
$$

with $n=1, \beta=21.8^{\circ}, n_{0}=\cos (\beta), \lambda=0.832, k_{0}=2 \pi / \lambda$. For the integration in time we have used in all cases the implicit midpoint rule (IMPR). We consider the initial condition

$$
u_{0}(x)=u_{0}^{1}(x)+u_{0}^{2}(x)+u_{0}^{3}(x)
$$

where

$$
u_{0}^{j}(x)=\exp \left(-\left(\left(x-\tau_{j}\right) / \nu\right)^{2}\right) \exp \left(i \eta_{j}\left(x-\tau_{j}\right)\right), \quad x \in[0, L],
$$

with $\eta_{j}=-\cos (\beta) k_{0} \tan \left(\alpha_{j}\right)$. Each $u_{0}^{j}(x)$ is a wave centered at $x=\tau_{j}$ and gives rise to a component of the solution that travels describing an angle $\alpha_{j}$ with the $t$-axis. In Figure 2 we have taken $\nu=10, \alpha_{1}=25^{\circ}, \alpha_{2}=17.8^{\circ}, \alpha_{3}=10^{\circ}, \tau_{1}=55, \tau_{2}=100$, $\tau_{3}=145$ and $L=200$. Therefore the solution consists of three components that arrive at the boundary $x=200$ at the same time. In Figure 2 we see the results when we consider $\mathrm{PF}(3,2)$ and the six nodes are chosen in the sense of least squares previously explained with $\rho=10,20,400$. We have draw in each case the relative $L^{2}$ error of the numerical solution (notice that for this kind of initial conditions the exact solution can be calculated explicitly and therefore we can measure this error). The six chosen nodes are optimal to absorb six concrete velocities $v_{j}=\tan \left(\beta_{j}\right)$. In the case of $\rho=10$ the angles $\beta_{j}$ are distributed in $\left(0,24.8^{\circ}\right)$ approximately. For $\rho=20, \beta_{j} \in\left(0,32.54^{\circ}\right)$ and for $\rho=400, \beta_{j} \in\left(0,70.87^{\circ}\right)$. Notice that the error for $t<350$ approximately is the same is each case since till that time the solution is traveling through the interior domain and it has not arrived at the boundary yet. That is, till that time the error is caused by the integration in the interior domain and does not depend on the $\mathrm{ABC}$ we are using. In this work we are interested in the error caused by the different $\mathrm{ABC}$ considered when the solution reaches the boundary (for $t>350$ approximately in this example). We observe that the best

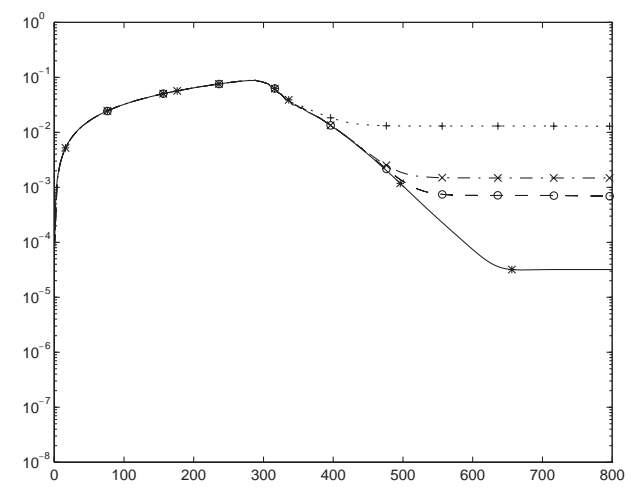

FiguRE 2. Error as a function of time. IMPR, $h=2.5 d-2$, $k=0.2$. $-*$ for adaptive $\operatorname{SABC}(3,2) ;--\circ$ for $\operatorname{PF}(3,2)$ when $\rho=10$; $-\cdot \times$ for $\operatorname{PF}(3,2)$ when $\rho=20$; and $\cdots+$ for $\operatorname{PF}(3,2)$ when $\rho=400$. 


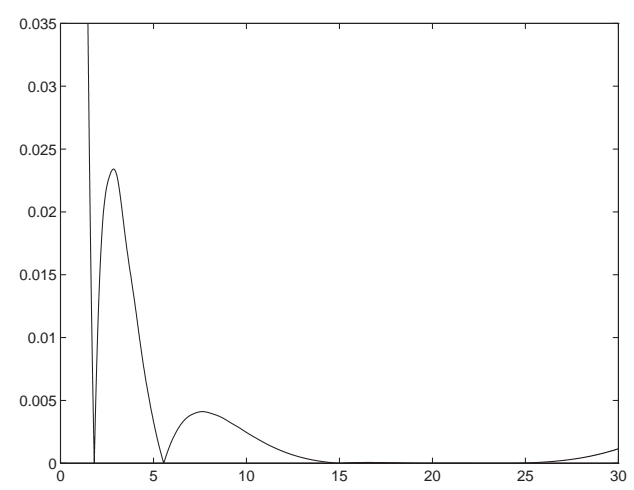

Figure 3. Reflection coefficient $\max \left(\left|K_{1}\right|,\left|K_{2}\right|\right)$ as a function of $\alpha$ for $\operatorname{PF}(3,2)$ when $\rho=10$.

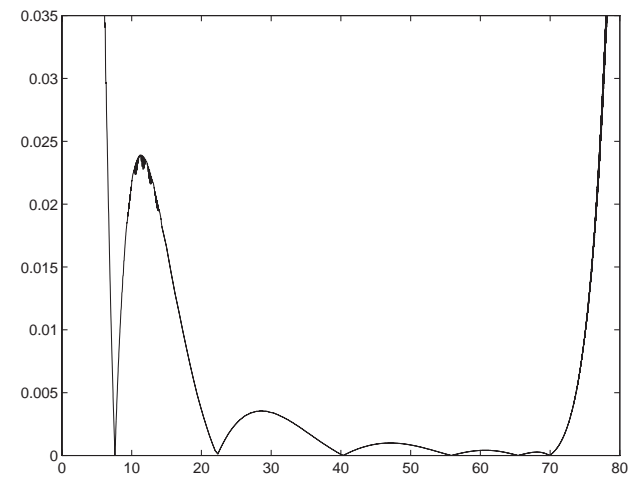

Figure 4. Reflection coefficient $\max \left(\left|K_{1}\right|,\left|K_{2}\right|\right)$ as a function of $\alpha$ for $\operatorname{PF}(3,2)$ when $\rho=400$.

result is obtained when $\rho=10$ and the worst with $\rho=400$. Let us explain the reason.

In [3] we measure the absorption of the boundary condition by the reflection coefficients $K_{1}, K_{2}$. The optimal situation is when $K_{1} \equiv K_{2} \equiv 0$ and then the solution is fully absorbed. This is the case of TBC. Nevertheless, with ABC the reflection coefficients only vanish at certain points which means that the $\mathrm{ABC}$ will absorb the velocities associated to those points. In Figure 3 we observe $\max \left(\left|K_{1}\right|,\left|K_{2}\right|\right)$ as a function of $\alpha$ for $\operatorname{PF}(3,2)$ when the nodes are chosen in $(0, \rho)$ with $\rho=10$. In this way, when $\max \left(\left|K_{1}\right|,\left|K_{2}\right|\right)=0$ at $\alpha=\alpha_{j}$, then this means that $\operatorname{PF}(3,2)$ will absorb the components of the solution traveling with velocity $\tan \left(\alpha_{j}\right)$. A similar picture can be seen in Figure 4 when $\rho=400$. The solution of the numerical experiment of Figure 2 has three principal components traveling at velocities $\tan \left(\alpha_{j}\right)$ for $\alpha_{j}=10^{\circ}, 17.8^{\circ}, 25^{\circ}$. Notice that the reflection coefficients for this values of $\alpha$ are much bigger in the case $\rho=400$ than when $\rho=10$. This is the reason why in Figure 2 the result is better when we consider $\rho=10$. 


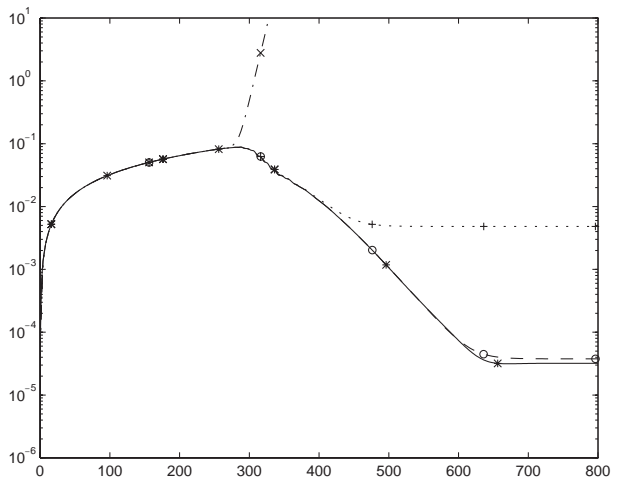

FiguRE 5. Error as a function of time. IMPR, $h=2.5 d-2$, $k=0.2$. $-*$ for adaptive $\operatorname{SABC}(3,2),--\circ$ for $\operatorname{PF}(4,3)$ when $\rho=10$, $-\cdot \times$ for $\operatorname{PF}(4,3)$ when $\rho=20$, and $\cdots+$ for $\operatorname{PF}(4,3)$ when $\rho=400$.

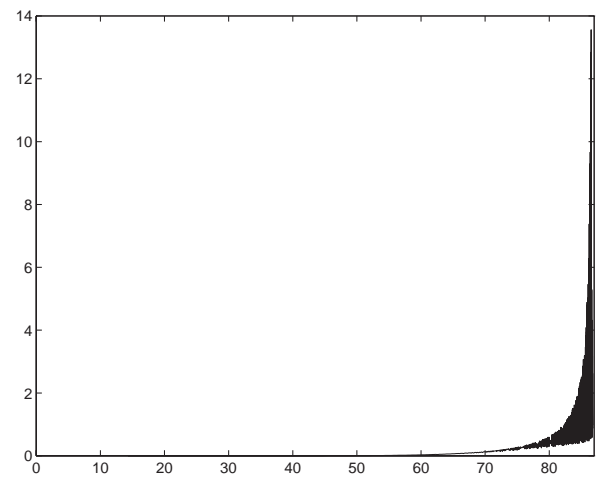

Figure 6. Reflection coefficient $\max \left(\left|K_{1}\right|,\left|K_{2}\right|\right)$ as a function of $\alpha$ for $\operatorname{PF}(4,3)$ when $\rho=20$.

Let us consider now $\mathrm{PF}(4,3)$, choosing eight nodes in $(0, \rho)$. We see in Figure 5 that when $\rho=20$, the error begins to grow. The absorption of the solution is not enough to compensate for the weak ill-posedness of the problem. The eight chosen nodes are optimal to absorb eight velocities $v_{j}=\tan \left(\beta_{j}\right)$ with the angles $\beta_{j}$ distributed in $\left(0,32.54^{\circ}\right)$ approximately. Nevertheless, the absorption of other velocities can be very poor and in the solution there can be components traveling with any velocity. Observe in Figure $6 \max \left(\left|K_{1}\right|,\left|K_{2}\right|\right)$ as a function of the $\alpha$ for all possible angles. Notice that the reflection coefficient is very big for large values of $\alpha$.

\section{Adaptive implementation}

In view of the disadvantages of considering fixed nodes, we have developed an adaptive implementation. We are going to modify the nodes so that they are 
suitable to absorb the numerical solution that is arriving at the boundary at each step in time.

Let us assume we have computed the numerical solution at time $t$, that is, we have $u^{j}(t), j=0, \ldots, N$. Let us consider first the left boundary with $\operatorname{SABC}\left(j_{1}, j_{2}\right)$ with $p=j_{1}+j_{2}+1$. At this point we want to choose $p$ suitable nodes $s_{j}, j=1, \ldots, p$, or what is the same after the change of variable (2.9), $\theta_{j}, j=1, \ldots, p$. Given the values of the numerical solution at $2 p$ points close to the boundary $u^{n}(t), n=1, \ldots, 2 p$, we are going to obtain the values of $\gamma_{k}, \tau_{k}$, $k=1, \ldots, p$, such that

$$
u^{n}(t) \approx \sum_{k=1}^{p} \gamma_{k} \exp \left(i\left(\tau_{k} n h-\omega\left(\tau_{k} h\right) t\right)\right), \quad n=1, \ldots, p .
$$

That is to say, we approximate $u^{n}(t)$ by means of a sum of plane waves. In order to obtain a good approximation, a possibility is to minimize the squared error over the data. However, this turns out to be a very expensive nonlinear problem. As an alternative, we have used Prony's algorithm [15], which permits us to obtain a good approximation, reducing the nonlinearity into a factoring of a polynomial of degree $p$.

The following step is to choose the interpolatory nodes in a suitable way in order to absorb as much as possible the solution arriving at the boundary. Since each $\tau_{k} h$ is associated to a velocity at which a component of the numerical solution is arriving at the boundary and we have $p$ nodes to be chosen, we could choose each one associated to one of the velocities calculated. Nevertheless, we should also take into account the amplitudes $\gamma_{k}, k=1, \ldots, p$. The idea is that if we have found a velocity with a much bigger amplitude than the rest, we should use more than one node to absorb the component of the numerical solution traveling at that velocity. To solve this problem, we have developed the following algorithm. For $j=1, \ldots, p$, if

$$
\left|\gamma_{k(j)}\right|=\max \left\{\left|\gamma_{1}\right|, \ldots,\left|\gamma_{p}\right|\right\},
$$

then we choose the node $\theta_{j}=\tau_{k(j)} h$ and we make $\gamma_{k(j)}=\gamma_{k(j)} / \sigma$ (in the numerical experiments, we have taken $\sigma=10$ ) and we start again until all the nodes are shared out. In this way, a velocity with a bigger amplitude is absorbed using more interpolatory nodes.

Let us carry out some numerical experiments showing the efficiency of this adaptive implementation. As in the previous section, in Figure 7 we consider the Fresnel equation (3.2) with initial condition

$$
u_{0}(x)=u_{0}^{1}(x)+\cdots+u_{0}^{6}(x),
$$

where $u_{0}^{j}(x)$ is given by (3.3) with $\alpha_{1}=17^{\circ}, \alpha_{2}=-30^{\circ}, \alpha_{3}=22^{\circ}, \alpha_{4}=-25^{\circ}$, $\alpha_{5}=27^{\circ}, \alpha_{6}=-20^{\circ}, \tau_{1}=100, \tau_{2}=140, \tau_{3}=180, \tau_{4}=220, \tau_{5}=260, \tau_{6}=300$, $\nu=10$ and $L=400$. That is, the solution consists of six components, three of which travel to the right and three to the left. For the integration in time we have used a diagonally implicit Runge-Kutta method of order 4 (DIRK4) which allows us to use bigger step sizes in time than the implicit midpoint rule. This fact will be especially important for the next experiments shown in Figures 8 , 9 and [10] where the solution travels with high velocity and otherwise a very small step size in time should be taken to integrate the problem, increasing in this way the computational cost. We observe in Figure 7 the relative error when we consider 


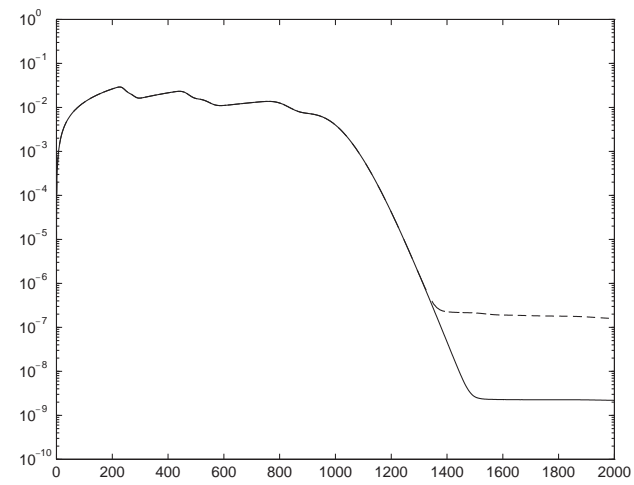

FiguRE 7. Error as a function of time. DIRK $4, h=1.25 d-2$, $k=2.5 d-2$. - for adaptive $\operatorname{SABC}(3,2) ;--$ for adaptive $\operatorname{SABC}(2,1)$.
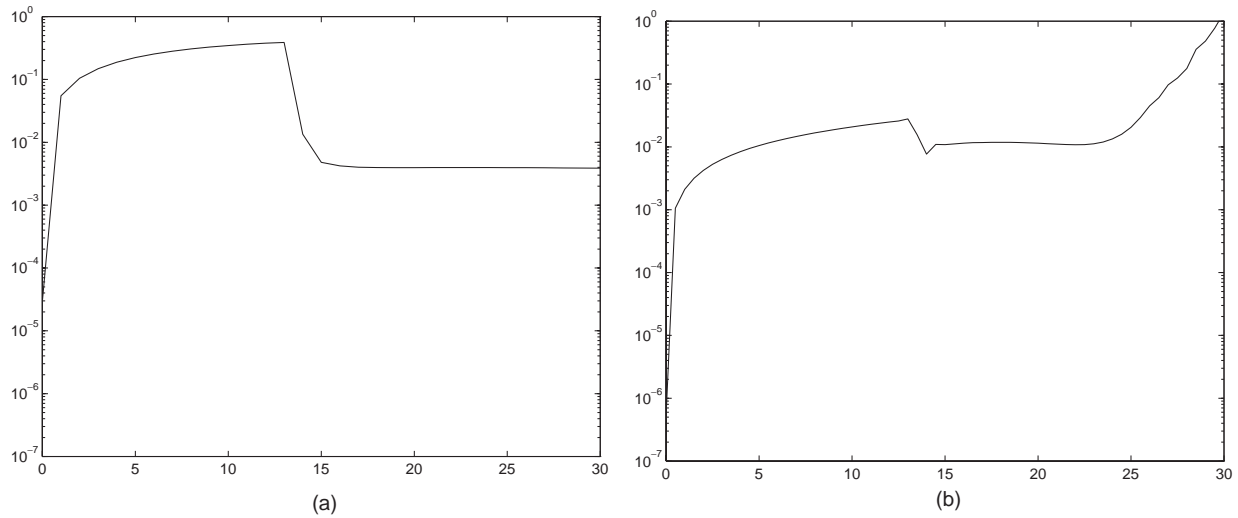

Figure 8. Error as a function of time. DIRK4. Adaptive $\operatorname{SABC}(3,2)$, (a) $h=1.0 d-3, k=5.0 d-4 ;$ (b) $h=2.5 d-4$, $k=2.5 d-4$.

$\operatorname{SABC}(3,2)$ and $\operatorname{SABC}(2,1)$ with the adaptive implementation previously explained. A great absorption is obtained due to the fact that the boundary conditions change automatically when each component of the solutions arrives at the boundary. Notice that the absorption is similar to that obtained in [3] for the case when the solution has a unique known group velocity and the SABC is fixed a priori in an optimal way to absorb it.

Let us consider the more difficult situation of the experiments of the previous section, where the three principal components of the solution, traveling with different velocities, arrive at the boundary at the same time. In Figures 2 and 5 we can see the result with $\operatorname{SABC}(3,2)$. We see that the absorption with the adaptive implementation is at least as good as with the nonadaptive one in any case. Moreover, we see in Figure 5 that the instability due to the small absorption of the nonadaptive implementation is avoided. 

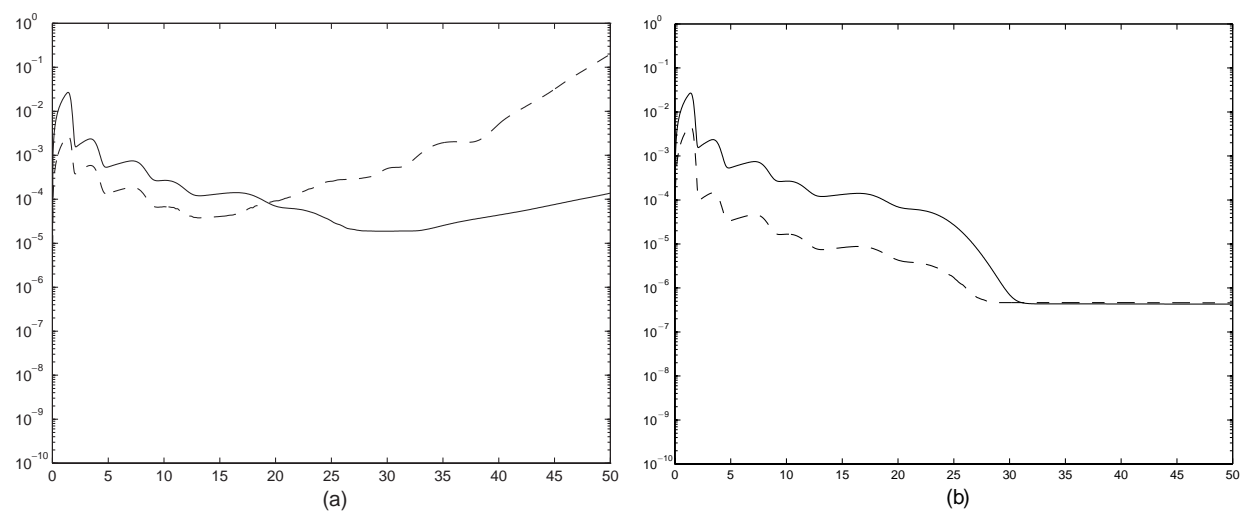

Figure 9. Error as a function of time. DIRK4. (a) Adaptive $\operatorname{SABC}(3,2):-$ for $h=5.0 d-4, k=2.5 d-4$ and -- for $h=2.5 d-4$, $k=2.5 d-4$. (b) Adaptive $\operatorname{SABC}(2,1)$ : - for $h=5.0 d-4$ and $k=2.5 d-4,--$ for $h=1.25 d-4, k=2.5 d-4$.

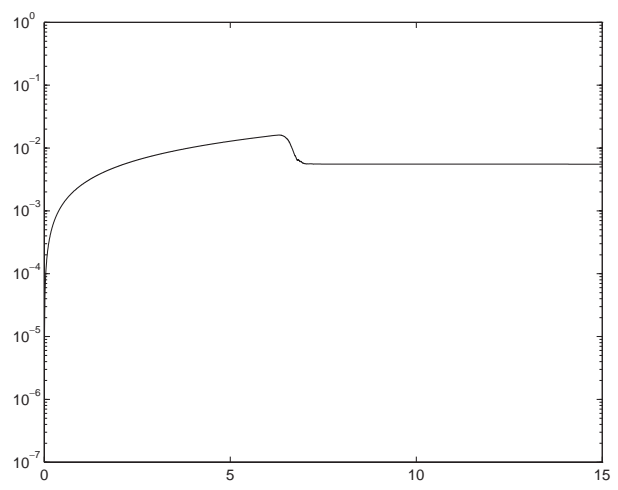

Figure 10. Error as a function of time. DIRK4. Adaptive $\operatorname{SABC}(2,1): h=2.5 d-4, k=2.5 d-4$. Initial condition $u_{0}(x)=u_{0}^{1}(x)+\cdots+u_{0}^{4}(x)$ with $\nu=3, \alpha_{1}=-70^{\circ}, \alpha_{2}=-75^{\circ}$, $\alpha_{3}=-80^{\circ}, \alpha_{4}=-85^{\circ}, \tau_{1}=18, \tau_{2}=24, \tau_{3}=37, \tau_{4}=75, L=93$.

Our next objective is to consider harder numerical experiments in order to see the limitations of $\operatorname{SABC}\left(j_{1}, j_{2}\right)$ with this new implementation. For this, let us consider now $\operatorname{SABC}(3,2)$, taking as initial condition

$$
u_{0}(x)=u_{0}^{1}(x)+\cdots+u_{0}^{6}(x),
$$

where $u_{0}^{j}(x)$ is given by (3.3) with $\nu=3, \alpha_{1}=85^{\circ}, \alpha_{2}=80^{\circ}, \alpha_{3}=75^{\circ}, \alpha_{4}=70^{\circ}$, $\alpha_{5}=60^{\circ}, \alpha_{6}=50^{\circ}, \tau_{1}=17, \tau_{2}=94, \tau_{3}=120, \tau_{4}=133, \tau_{5}=147, \tau_{6}=154$ and $L=170$, so that the solution consists of six waves with different velocities (some of them quite high) that arrive at the boundary at the same time. Since for $\operatorname{SABC}(3,2)$ we have six nodes to absorb the solution, for this experiment, the adaptive implementation is supposed to use only one node to absorb each velocity that arrives at the same time at the boundary. This is why in Figure 8 (a) the 
absorption is much smaller than in the previous numerical experiments. One of the difficulties when integrating waves that travel with a high velocity is that the step sizes, both in time and space, should be taken very small so that the error committed in the interior domain is small. In Figure 8 (a) we have taken $h=1.0 d-3$, $k=5.0 d-4$, using DIRK4 for the integration in time. Nevertheless, as with these values of $h, k$, the error in the interior domain is too big; in Figure 8 (b) we have reduced the stepsizes to $h=2.5 d-4, k=2.5 d-4$. Now the error in the interior domain is smaller, but due to the reduction of $h$, we observe a growth of the error since the absorption is not enough to compensate for the weak ill-posedness of the semidiscrete problem for $\operatorname{SABC}(3,2)$ studied in [3].

When $h$ is chosen small enough, the problems of instability for $\operatorname{SABC}(3,2)$ can emerge in other kinds of experiments when there are not several waves arriving exactly at the same time at the boundary. This is seen in Figure 9a) where the initial condition

$$
u_{0}(x)=u_{0}^{1}(x)+\cdots+u_{0}^{6}(x),
$$

where $u_{0}^{j}(x)$ is given by (3.3) with $\nu=3, \alpha_{1}=-85^{\circ}, \alpha_{2}=-71^{\circ}, \alpha_{3}=-57^{\circ}$, $\alpha_{4}=50^{\circ}, \alpha_{5}=64^{\circ}, \alpha_{6}=78^{\circ}, \tau_{1}=18, \tau_{2}=23, \tau_{3}=28, \tau_{4}=33, \tau_{5}=38, \tau_{6}=43$ and $L=61$. This way, there are six waves arriving at both boundaries at different times. Nevertheless, notice that the wave with highest velocity has already been absorbed at $t \approx 2$, while the slowest one is absorbed at $t \approx 25$. Therefore, when the slowest wave arrives at the boundary, it does not arrive "alone", but with the reflections produced by the absorption of the other five waves. We see in Figure 9 a) that for $h=5.0 d-4, k=2.5 d-4$ there is a light growth of the relative error. This growth is increased if, for the same value of $k$, we take the smaller value $h=2.5 d-4$. This behavior is due to the weak ill-posedness of the semidiscrete problem for $\operatorname{SABC}(3,2)$.

Another option is to use $\operatorname{SABC}(2,1)$ for which the semidiscrete problems have a weaker ill-posedness. In fact, for $\operatorname{SABC}(3,2)$ we have the estimate $\kappa_{h} \approx O\left(h^{-3.5}\right)$ for the condition number of the matrix of eigenvectors, while for $\operatorname{SABC}(2,1), \kappa_{h} \approx$ $O\left(h^{-2}\right)$. In Figure 9(b) we have repeated the experiment of Figure 9(a) with $\operatorname{SABC}(2,1)$ and $h=5.0 d-4, k=2.5 d-4$ and we do not observe the bad behavior due to ill-posedness. Nevertheless, as the problem for $\operatorname{SABC}(2,1)$ is also weakly ill-posed, in order to see if a reduction of $h$ could lead to a growth of the error, we have considered the same experiment with the same value for $k$ and $h=1.25 d-4$ (notice that this value is smaller than both values of $h$ considered in Figure 9(a) for $\operatorname{SABC}(3,2))$. The numerical result indicates that the instability is not observed and with approximately the same final absorption, we obtain a smaller error in the interior domain.

Of course, we remark that the absorption for $\operatorname{SABC}(2,1)$ is smaller than for $\operatorname{SABC}(3,2)$. For instance, see Figure 7 when the components of the solution do not arrive simultaneously at the boundary, and Figure 10 when four waves arrive at the same time at the boundary so that $\operatorname{SABC}(2,1)$ can only assign one interpolatory node to absorb each wave.

\section{Appendix A. Formulas for the COEFficients of SABC}

In this appendix we present the coefficients of the rational functions $q(s, t)$ considered to obtain $\operatorname{SABC}(2,1), \operatorname{SABC}(3,2)$ and $\operatorname{SABC}(4,3)$. 
$\operatorname{SABC}(\mathbf{2}, \mathbf{1})$. This $\mathrm{SABC}$ is obtained when we consider

$$
q(s, h)=\frac{\alpha_{0}+\alpha_{1} s+\alpha_{2} s^{2}}{1+\alpha_{3} s}
$$

that interpolates $r(s, h)$ at $s_{j}$ for $j=1, \ldots, 4$. We have obtained that

$$
\begin{aligned}
& \alpha_{0}=1+\frac{2 i d_{4,4}}{d_{4,3}}, \\
& \alpha_{1}=\frac{h^{2}\left(d_{4,1}+2 i d_{4,2}-3 d_{4,3}-4 i d_{4,4}\right)}{4 d_{4,3}}, \\
& \alpha_{2}=\frac{h^{4}\left(i-d_{4,1}-i d_{4,2}+d_{4,3}+i d_{4,4}\right)}{8 d_{4,3}}, \\
& \alpha_{3}=\frac{h^{2}\left(d_{4,1}-d_{4,3}\right)}{4 d_{4,3}}
\end{aligned}
$$

where

$$
d_{n, j}=\sum_{r_{j} \in C_{n, j}} \pi r_{j}, \quad j=1, \ldots, n,
$$

where $C_{n, j}$ is the set of combinations of $j$ elements of the set $\left\{t_{1}, \ldots, t_{n}\right\}$ and $t_{k}$ and $s_{k}$ are related through the changes of variables (2.9) $-(2.10)$. We have used the notation $\pi r_{j}$ for the product of the elements of $r_{j} \in C_{n, j}$.

$\mathbf{S A B C}(\mathbf{3 , 2})$. In this case, the rational function considered is

$$
q(s, h)=\frac{\alpha_{0}+\alpha_{1} s+\alpha_{2} s^{2}+\alpha_{3} s^{3}}{1+\alpha_{4} s+\alpha_{5} s^{2}}
$$

that interpolates $r(s, h)$ at $s_{j}$ for $j=1, \ldots, 6$. The coefficients in this case are

$$
\begin{aligned}
& \alpha_{0}=1+\frac{2 i d_{6,6}}{d_{6,5}}, \\
& \alpha_{1}=\frac{h^{2}\left(d_{6,3}+2 i d_{6,4}-4 d_{6,5}-6 i d_{6,6}\right)}{4 d_{6,5}}, \\
& \alpha_{2}=\frac{h^{4}\left(d_{6,1}+2 i d_{6,2}-3 d_{6,3}-4 i d_{6,4}+5 d_{6,5}+6 i d_{6,6}\right)}{16 d_{6,5}}, \\
& \alpha_{3}=\frac{h^{6}\left(i-d_{6,1}-i d_{6,2}+d_{6,3}+i d_{6,4}-d_{6,5}-i d_{6,6}\right)}{32 d_{6,5}}, \\
& \alpha_{4}=\left(\frac{h^{2}}{2}\right)\left(\frac{d_{6,3}}{2 d_{6,5}}-1\right) \\
& \alpha_{5}=\frac{h^{4}\left(d_{6,1}-d_{6,3}+d_{6,5}\right)}{16 d_{6,5}},
\end{aligned}
$$

with $d_{6, j}$ given by (A.1).

SABC $(4,3)$. The rational function considered in this case is

$$
q(s, h)=\frac{\alpha_{0}+\alpha_{1} s+\alpha_{2} s^{2}+\alpha_{3} s^{3}+\alpha_{4} s^{5}}{1+\alpha_{5} s+\alpha_{6} s^{2}+\alpha_{7} s^{3}}
$$


that interpolates $r(s, h)$ at $s_{j}$ for $j=1, \ldots, 8$. The coefficients in this case are

$$
\begin{aligned}
& \alpha_{0}=1+\frac{2 i d_{8,8}}{d_{8,7}}, \\
& \alpha_{1}=\frac{h^{2}\left(d_{8,5}+2 i d_{8,6}-5 d_{8,7}-8 i d_{8,8}\right)}{4 d_{8,7}}, \\
& \alpha_{2}=\frac{h^{4}\left(d_{8,3}+2 i d_{8,4}-4 d_{8,5}-6 i d_{8,6}+9 d_{8,7}+12 i d_{8,8}\right)}{16 d_{8,7}}, \\
& \alpha_{3}=\frac{h^{6}\left(d_{8,1}+2 i d_{8,2}-3 d_{8,3}-4 i d_{8,4}+5 d_{8,5}+6 i d_{8,6}-7 d_{8,7}-8 i d_{8,8}\right)}{64 d_{8,7}}, \\
& \alpha_{4}=\frac{h^{8}\left(i-d_{8,1}-i d_{8,2}+d_{8,3}+i d_{8,4}-d_{8,5}-i d_{8,6}+d_{8,7}+i d_{8,8}\right)}{128 d_{8,7}}, \\
& \alpha_{5}=\frac{h^{2}}{2}\left(\frac{-3}{2}+\frac{d_{8,5}}{2 d_{8,7}}\right), \\
& \alpha_{6}=\frac{h^{4}\left(d_{8,3}-2 d_{8,5}+3 d_{8,7}\right)}{16 d_{8,7}}, \\
& \alpha_{7}=\frac{h^{6}\left(d_{8,1}-d_{8,3}+d_{8,5}-d_{8,7}\right)}{64 d_{8,7}},
\end{aligned}
$$

with $d_{8, j}$ given by (A.1).

\section{REFERENCES}

[1] I. Alonso-Mallo and N. Reguera, Condiciones frontera transparentes y absorbentes para la ecuación de Schrödinger en una dimensión, Proceedings of the 16th Congress on Differential Equations and Applications and 6th Congress on Applied Mathematics, Las Palmas de Gran Canaria, Spain, 1999, 467-473.

[2] I. Alonso-Mallo and N. Reguera, Weak ill-posedness of spatial discretizations of absorbing boundary conditions for Schrödinger-type equations, SIAM J. Numer. Anal., 40, (2002), 134158.

[3] I. Alonso-Mallo and N. Reguera, Discrete absorbing boundary conditions for Schrödinger-type equations. Construction and error analysis, to appear in SIAM J. Numer. Anal.

[4] B. Alpert, L. Greengard and T. Hagstrom, Rapid evaluation of nonreflecting boundary kernels for time-domain wave propagation, SIAM J. Numer. Anal., 37, (2000), 1138-1164. MR 2002c: 65037

[5] V. A. Baskakov and A. V. Popov, Implementation of transparent boundaries for numerical solution of the Schrödinger equation, Wave Motion, 14, (1991), 123-128. MR 92g:78001

[6] L. Di Menza, Transparent and absorbing boundary conditions for the Schrödinger equation in a bounded domain, Numer. Funct. Anal. and Optimiz., 18, (1997), 759-775. MR 98h:65036

[7] E. Dubach, Artificial boundary conditions for diffusion equations: Numerical study, J. Comp. App. Math., 70, (1996), 127-144. MR 97c:65211

[8] T. Fevens and H. Jiang, Absorbing boundary conditions for the Schrödinger equation, SIAM J. Sci. Comput., 21, (1999), 255-282. MR 2000h:65144

[9] L. Halpern, Absorbing boundary conditions for the discretization schemes of the onedimensional Wave equation, Math. Comput., 38, (1982), 415-429. MR 83d:65245

[10] L. Halpern and J. Rauch, Absorbing boundary conditions for diffusion equations, Numer. Math., 71, (1995), 185-224. MR 96h:65152

[11] L. Halpern and L.N. Trefethen, Wide-angle one-way equations, J. Acoust. Soc. Am., 84, (1988), 1397-1404. MR 89j:76076

[12] R.L. Higdon, Radiation boundary conditions for dispersive waves, SIAM J. Numer. Anal. 31, (1994), 64-100. MR 94k:35178

[13] W. Huang, C. Xu, S. Chu and S. Chaudhuri, The Finite-Difference Vector Beam Propagation Method: Analysis and Assesment, J. Lightwave Techn., 10, (1992), 295-305. 
[14] C. Lubich and A. Schädle, Fast convolution for non-reflecting boundary conditions, SIAM J. Sci. Comput., 21, (2002), 161-182.

[15] S. L. Marple Jr., Digital Spectral Analysis with applications, Prentice Hall, Englewood Cliffs, 1987. MR 89c:93001

[16] A. Messiah, Quantum Mechanichs, Vol.1, trans. G. Temmer, Interscience, New York, 1961. MR 23:B2826

[17] N. Reguera, Stability of a class of matrices with applications to absorbing boundary conditions for Schrödinger-type equations, to appear in Appl. Math. Lett.

[18] N. Reguera, Analysis of a third order absorbing condition for the Schrödinger equation discretized in space, to appear in Appl. Math. Lett.

[19] F. Schmidt, An adaptive approach to the numerical solution of Fresnel's wave equation, J. Lightwave Technol., 11, (1993), 1425-1434.

[20] F. Schmidt and D. Yevick, Discrete transparent boundary conditions for Schrödinger-type equations, J. Comp. Phys., 134, (1997), 96-107. MR 98e:81028

Departamento de Matemática Aplicada y Computación, Universidad de Valladolid, VALLADOLID, SpAin

E-mail address: isaias@mac.cie.uva.es

Departamento de Matemáticas y Computación, Universidad de Burgos, Burgos, Spain

E-mail address: nreguera@ubu.es 